\title{
Diccionario histórico-toponímico de la Isla del Coco, Costa Rica
}

\author{
Ronald Eduardo Díaz Bolaños ${ }^{1,2}$, Eric J. Alfaro ${ }^{1,3,4}$ \& Alexia Ugalde Quesada ${ }^{1}$ \\ 1. Centro de Investigaciones Geofísicas (CIGEFI), Ciudad de la Investigación, Universidad de Costa Rica, San Pedro, \\ 11501-2060 San José, Costa Rica. \\ 2. Cátedra de Historia, Universidad Estatal a Distancia, San José, Costa Rica. \\ 3. Centro de Investigación en Ciencias del Mar y Limnología (CIMAR), Universidad de Costa Rica. \\ 4. Escuela de Física, Universidad de Costa Rica.
}

Recibido 02-II-2012. Corregido 26-VII-2012. Aceptado 24-IX-2012.

\begin{abstract}
Historic-Toponymic dictionary for Isla del Coco, Costa Rica. Many names have been used for the different places of Isla del Coco, and some have changed over time. Studying different historic sources, seventy-six names of the main topographic features were collected for Isla del Coco National Park, Costa Rica. These names could be grouped in four main categories: those associated with the British traveler's visitations, the ones related with the republican period of Costa Rica and those linked with the island's terrestrial and marine flora and fauna. The methodology used allowed the proper documentation of seventy-one names. This methodology and the publication of this dictionary intend to generate a feedback regarding the naming of topographic characters of Isla del Coco. Citation: Díaz-Bolaños, R.D., E.J. Alfaro \& A. Ugalde-Quesada. 2012. Diccionario histórico-toponímico de la Isla del Coco, Costa Rica. Rev. Biol. Trop. 60 (Suppl. 3): 1-14. Epub 2012 Dec 01.
\end{abstract}

Key words: Isla del Coco, Cocos Island, eastern tropical Pacific, Costa Rican history, toponymy, Central America, map.

La Isla del Coco es un territorio insular costarricense que se ubica en las aguas del Océano Pacífico, a $5^{\circ} 32^{\prime} \mathrm{N}$ y a $87^{\circ} 04^{\prime} \mathrm{W}$, posee una superficie de $24 \mathrm{~km}^{2}$ y es la porción del territorio costarricense más próxima a la línea ecuatorial. Esta isla se caracteriza por ser el único afloramiento de la Cordillera Volcánica del Coco, alineamiento volcánico situado sobre la placa homónima que se levanta hasta 3000 $\mathrm{m}$ del fondo del océano. Esta isla está cubierta principalmente por bosques tropicales y su topografía es bastante irregular, tanto en la línea costera como en su interior (Boza 1984, Weston 1992). Esta es la única isla oceánica de América Central y se caracteriza por su gran biodiversidad, tanto en la sección terrestre como en la marítima por la presencia de importantes arrecifes coralinos y otros ambientes marinos (Guzmán \& Cortés 1992, 1993, 2007, Carbonell 1998, Fernández 2008).

La primera exploración de la isla tuvo lugar hacia mediados de la década de 1520 por parte del capitán español Juan Cabezas (siglo XVI) y dada su ubicación, fue visitada por numerosos buques en los siglos siguientes. La isla se convirtió en un refugio para piratas que merodeaban la costa pacífica de las posesiones españolas en América, por lo que su nombre se asocia a la existencia de tres supuestos tesoros que fueron abandonados en ella en los años 1685,1820 y 1821, razón por las que se organizaron numerosas expediciones para determinar su ubicación y extraerlos (Boza 1984, Arias 1993).

El gobierno de Costa Rica, tomó formalmente posesión de la Isla del Coco el 15 de setiembre de 1869 , por medio de una expedición dirigida por Rafael Oreamuno. En los años siguientes, la isla albergó temporalmente un presidio y fue objeto de un fallido intento de colonización agrícola liderado por el alemán August Gissler (1857-1935). La soberanía costarricense sobre la isla fue ratificada durante la visita del presidente Rodrigo Carazo 
(1926-2009) el 24 de junio de 1978 y administrativamente constituye el distrito décimo del cantón de Puntarenas (Boza 1984, Weston 1992, Arias 1993, Ministerio de Obras Públicas y Transportes 2009).

Desde un punto de vista geoestratégico, la isla se encuentra en un sitio idóneo para el control de rutas marítimas e incursiones militares en el Océano Pacífico oriental, por lo que en 1938, tres años después de la visita del presidente estadounidense Franklin D. Roosevelt (1882-1945) a la isla, el gobierno costarricense propuso al de los Estados Unidos la posibilidad de ceder el territorio insular para una base naval, hecho que no sucedió por el interés norteamericano en las Islas Galápagos que presentaba mejores condiciones para la instalación de una base militar que funcionó durante la Segunda Guerra Mundial (1939-1945) (Sylva, 1992, Weston 1992, Steinleger 2004, Segura 2006, Reinoso, com. pers. 2012).

La Isla del Coco ha recibido algunas distinciones, entre ellas la declaratoria de Parque Nacional por medio del Decreto Ejecutivo $\mathrm{N}^{\circ}$ 8748-A del 22 de junio de 1978, que fue reafirmada con la promulgación de la Ley N ${ }^{\circ} 6794$ del 25 de agosto de 1982. En 1997, la Organización de las Naciones Unidas para la Educación y la Cultura (UNESCO) la incluyó en la lista de sitios considerados Patrimonio Natural de la Humanidad y en 1998, se le reconoció como humedal de importancia internacional según la Convención de Humedales de Ramsar (Boza 1984, Carbonell 1998). El 11 de octubre de 2002, se le declara monumento histórico por la presencia de numerosos vestigios presentes en la isla que atestiguan el constante arribo de visitantes desde tiempos coloniales hasta finales del siglo XX (Cortés 2008, ICOMOS Costa Rica, sf).

La Isla del Coco ha sido objeto de diversas expediciones científicas efectuadas desde finales del siglo XVII hasta el presente, que además de efectuar misiones de reconocimiento geográfico y de recolección de muestras de flora y fauna, se han dedicado también a evaluar el potencial económico de su territorio (Cortés 2008). Fruto de esas exploraciones ha sido un importante corpus cartográfico y la publicación de varios libros y artículos en revistas científicas, por lo que los nombres de muchos de los accidentes geográficos están relacionados con estas investigaciones.

Debido a lo anterior, se plantea como objetivo general de este trabajo el elaborar un diccionario histórico-toponímico que explique el origen de los nombres de los accidentes geográficos presentes en la Isla del Coco, a partir de la investigación de las fuentes documentales relacionadas con las expediciones que visitaron la isla entre los siglos XVI y XX, del que proceden los nombres de sus geoformas y sitios más representativos. Precisamente, la toponimia comprende los nombres geográficos que evidencian la huella dejada por los seres humanos que ocuparon un espacio a través del tiempo (Moreno, s.f.).

La siguiente sección corresponde a la metodología empleada y las fuentes documentales, seguida de una síntesis de los antecedentes de las investigaciones en el campo de la toponimia en Costa Rica. Posteriormente, se describen ejemplos sobre los principales resultados y su discusión.

\section{METODOLOGÍA Y FUENTES DOCUMENTALES}

La base de la investigación del presente proyecto consiste en una revisión bibliográfica tal y como lo sugiere Gallardo (1991), en la que se recopilaron varios textos publicados en revistas, folletos o libros que se encuentran disponibles en el Archivo Nacional de Costa Rica (ANCR), la Biblioteca Nacional de Costa Rica Miguel Obregón Lizano (BNMOL) y las Bibliotecas Carlos Monge Alfaro, Luis Demetrio Tinoco Castro y de la Facultad de Derecho de la Universidad de Costa Rica, además de algunos textos procedentes de la bibliotecas particulares (e.g. J. Cortés com. pers. 2010).

Los textos seleccionados fueron clasificados en fuentes primarias y secundarias de acuerdo con los criterios empleados por Gallardo (1991). Las fuentes primarias comprenden aquellas que brindan información de primera 
mano sobre los aspectos que se van a investigar, en este caso, los topónimos (nombres de lugares de la Isla del Coco), mientras que las secundarias ofrecen información reelaborada de las fuentes originales.

Algunas de las fuentes primarias incluyen: 1) Crónicas de viajeros (Dampier 1697, Wafer 1967); 2) Informes de expediciones científicas (Alfaro 1963, Biolley 1935, Chavarría 1963, Lièvre 1962, Passmore 1963, Pittier 1899); 3) Colección de Leyes, Decretos y Acuerdos de la República de Costa Rica; 4) Mapas (Figueroa 1900, Instituto Geográfico Nacional 2002, J. García, com. pers. 2010) y 5) Entrevistas y cuestionarios a personas que han trabajado en la isla (Cortés, com. pers.-cuestionario 2011, Herrera et al. com. pers.-cuestionario 2011, Montoya, com. pers. 2012).

Las fuentes secundarias incluyen: 1) Libros (p.e. Weston 1992), 2) artículos de revistas (p.e. Ancey 1903) y 3) Tesis (p.e. Arias 1993, 2004).

En las fuentes, tanto primarias como secundarias, se recopilan los términos geográficos y se asocian con los nombres de quienes participaron en las exploraciones científicas que visitaron la isla, así como de algunas figuras políticas que se relacionaron directa o indirectamente con ellas. En estos casos, se agrega información de datos históricos y biográficos referentes a estos actores.

Una vez seleccionada la información, esta se organiza alfabéticamente por medio de categorías referentes a los distintos tipos de accidentes geográficos. Además, siguiendo las clasificaciones propuestas por Azofeifa (1986) se han agrupado los topónimos a partir del motivo del nombre en epotopónimos (referencia a personajes), fisiotopónimos (características del mismo accidente), pragmatopónimos (conmemoración de hechos) y topotopónimos (procedente de otro topónimo) e incluso a partir de la naturaleza de los accidentes como los actiónimos (relacionados con sitios costeros). Además, se agregan los fitotopónimos cuando el nombre del lugar hace mención a la flora, los zootopónimos que se refieren a la fauna, los cronotopónimos al tiempo y los lembotopónimos cuando la geoforma recibe su nombre a partir de una embarcación que visitó la isla.

\section{ANTECEDENTES}

La toponimia ha sido investigada en Costa Rica desde finales del siglo XIX siendo el primer trabajo pionero la obra de Pittier (1895) al explorar el origen de los nombres de Talamanca. Gagini (1979) incluyó también explicaciones sobre la procedencia de varios topónimos mientras que Azofeifa (1986) analizó el origen del nombre de cada uno de los cantones y ofreció una clasificación según su procedencia.

Más recientemente se han elaborado investigaciones sobre la toponimia provincial de Costa Rica en los que se destacan el origen y la clasificación de los diversos nombres, así como explicaciones sobre su procedencia (Garita 1995, 1998, 2000, Garita y Cordero 2002, Monge et al. 2003, Chang 2010, Hernández y Bedoya, 2010). Sin embargo, estos trabajos no se han referido a la toponimia de la Isla del Coco, salvo el de Garita y Cordero (2002) que lo hace someramente cuando se refiere a la toponimia de la provincia de Puntarenas. Por eso, la presente investigación se dedica a analizar los topónimos de la Isla del Coco explicando su origen y clasificándolos según sus características.

\section{RESULTADOS}

A partir de los mapas consultados, se localizaron setenta y seis topónimos. Su clasificación se muestra en el Cuadro 1 y su ubicación en la Figura 1. Los topónimos se han clasificado en catorce categorías a partir de las características de cada uno de ellos (Cuadro 2). La décimo cuarta categoría corresponde a aquellos topónimos que no ha sido posible clasificar o documentar en el desarrollo actual de la investigación y de acuerdo con Montoya (com. pers. 2012) fueron asignados por los mismos funcionarios del parque nacional en períodos recientes y con excepción del Cerro Las Madres, podrían tratarse de epotopónimos.

A partir de las clasificaciones propuestas por Azofeifa (1986), los topónimos aparecen distribuidos en el Cuadro 3 según su tipo. Entre los topónimos investigados, se han 


\section{CUADRO 1}

Topónimos identificados en la Isla del Coco. Aquellos que no se pudieron documentar se muestran en itálica.

TABLE 1

Identified toponymys of Isla del Coco. Those that could not be documented are in italics.

\section{Topónimo}

Número

Bahías: Chatham, Iglesias (Yglesias) o Esperanza, Inútil, Wafer y Weston

5

Bajos: Alcyone, Dos Amigos y Punta María

Cabos: Atrevida, Barreto, Dampier o Esquina Manta, Descubierta, Discovery y Lionel 6

Cascadas: Iglesias (Yglesias)

Cerros: Jesús Jiménez, Iglesias (Yglesias), Pelón y Cerros Las Madres

Estrechos: Challe y Esperanza

Islas: Cáscara o Viquinga, Coco, Cónico, Dos Amigos, Manuelita o Nuez, Muela o Taza de Té, Pájara, Vancouver o Juan Bautista y Montagne

Llanuras: Llanos Palo de Hierro

Penínsulas: Colnett

Puntas: Agujas, Don Felipe, Don Will, Gissler, Madriz, María, Montealegre, Pacheco, Presidio, Quirós, Rodríguez, Sofía, Turrialba, Ulloa y El Coronel

Quebradas: 15 de Setiembre, Albatros, Alfaro, Alvarado, Camila, Camarón, El Anolís, El Cabro, El

Cuclillo, El Gato, El Pinzón, Escorpión, Espíritu Santo, Haug, Lièvre, Minuto, Pittier, Venado, Jordi y

Monserrat

Ríos: Genio e Iglesias (Yglesias)

Rocas: Aleta de Tiburón, Bote, Gissler, Rocas Barlovento, Pan de Azúcar, Sucia y Sumergida

Total

Fuente: García (s.f.) e Instituto Geográfico Nacional (2002).

CUADRO 2

Topónimos clasificados según características

TABLE 2

Toponymys classified by their characteristics

\begin{tabular}{lc}
\multicolumn{1}{c}{ Características de los topónimos } & Cantidad \\
Actores políticos & 12 \\
Científicos & 2 \\
Embarcaciones & 9 \\
Exploradores & 7 \\
Flora & 3 \\
Fauna marina & 1 \\
Fauna terrestre & 9 \\
Funcionarios & 6 \\
Geomorfología & 15 \\
Historia de la Isla del Coco & 2 \\
Navegantes & 3 \\
Tiempo & 1 \\
Visitantes & 1 \\
No clasificados o documentados & 5 \\
Total & 76
\end{tabular}

\section{CUADRO 3}

Topónimos clasificados según tipo

TABLE 3

Toponymys classified by their type

\begin{tabular}{lc}
\multicolumn{1}{c}{ Tipo de topónimos } & Cantidad \\
Actiónimos & 2 \\
Cronotopónimos & 1 \\
Epotopónimos & 31 \\
Fisiotopónimos & 12 \\
Fitotopónimos & 3 \\
Lembotopónimos & 9 \\
Pragmatopónimos & 2 \\
Topotopónimos & 1 \\
Zootopónimos & 10 \\
No clasificados o documentados & 5 \\
Total & 76
\end{tabular}




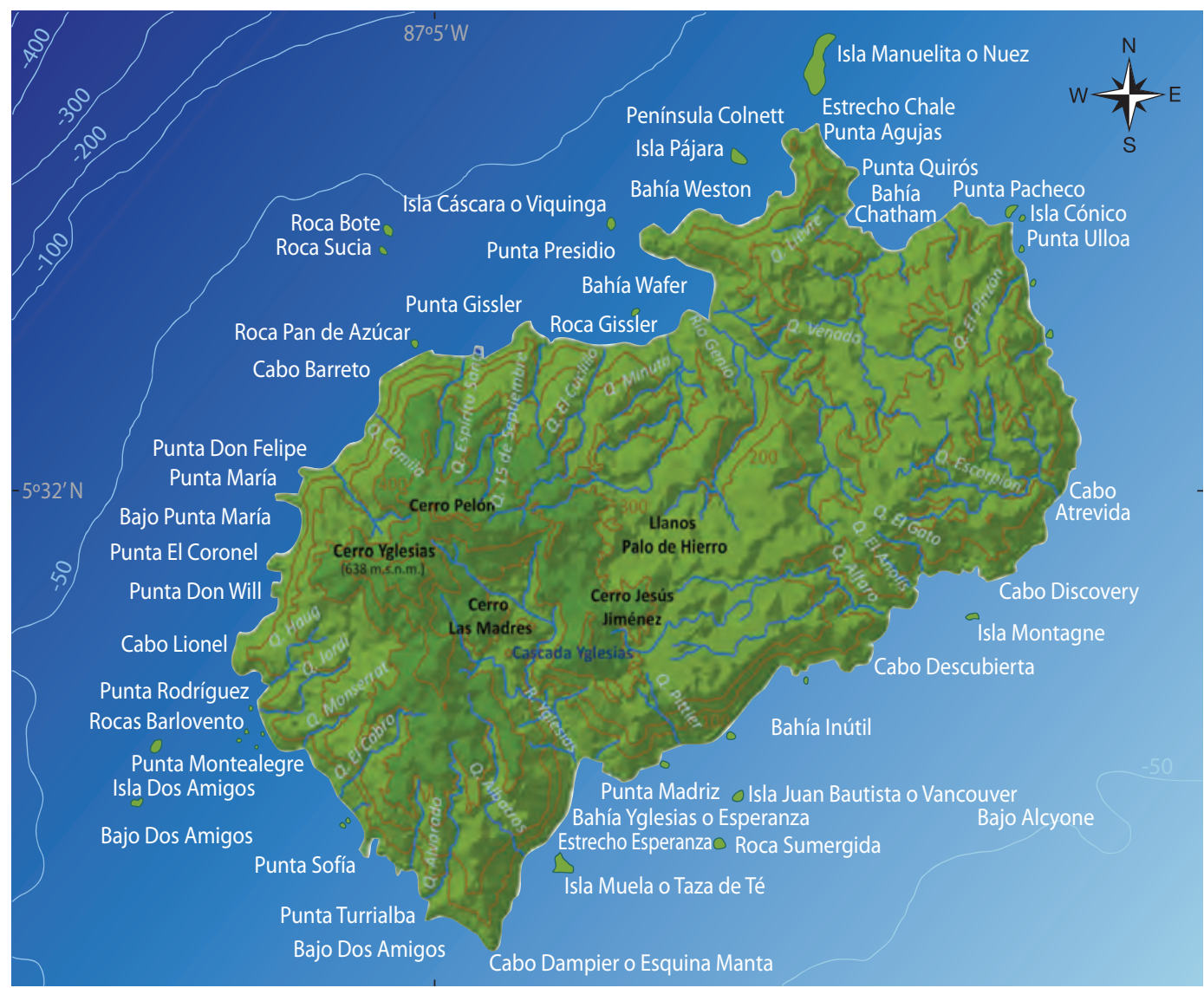

Fig. 1. Principales accidentes geográficos de la Isla del Coco, Costa Rica. Basado en el mapa de García (s.f.) e Instituto Geográfico Nacional (2002).

Fig. 1. Main geographic accidents of Isla del Coco, Costa Rica. Based on the map by García (no date) and Instituto Geográfico Nacional (2002).

seleccionado cinco de los que se ha recogido información para el diccionario y se explican a continuación a modo de ejemplo de la metodología empleada. En el anexo 1 se incluye una breve descripción de los topónimos cuyo origen ha sido identificado.

Un caso interesante de analizar es el nombre propio de la Isla del Coco. El nombre de la isla aparece por primera vez en el mapamundi de Enrique II de Francia (1542) con el nombre de Ysle de Coques. Este mapa fue dibujado por el cartógrafo francés Nicolás Desliens (siglo XVI) y representa las costas del continente americano reconocidas por los europeos. La palabra coque en francés significa "cáscara de nuez" por lo que posiblemente el nombre original era Isla Cáscara, término que se conserva en la toponimia de la zona con la pequeña isla del mismo nombre que se ubica frente a la punta Presidio en la Bahía Wafer (Lièvre 1962, Montoya com. pers. 2012). Además, frente a la Península de Colnett se encuentra la Isla Nuez, llamada también Manuelita, por lo que su nombre también constituye una reminiscencia del nombre original de la Isla del Coco. Cabe destacar que en la isla abunda el llamado Palo de Hierro (Sacoglottis holdridgei), cuya semilla semeja una nuez, y que le da su nombre a los llanos ubicados en el centro de la isla (Weston 1992, Montoya 2007). 
La isla también fue muy visitada por navegantes británicos, por ejemplo, la Bahía Wafer, principal punto de acceso a la isla, fue denominada en honor a Lionel Wafer (16401705), viajero y cirujano galés del siglo XVII que acompañó a William Dampier (1652-1715) en sus expediciones. Dampier es el nombre que recibe el cabo ubicado más al sur de la isla, también llamado Esquina Manta. Wafer fue el primero en describir las condiciones naturales de la Isla del Coco (Arias 1993). Por su parte, la Bahía Chatham recibe su nombre procedente de una de las embarcaciones que integraron la expedición de George Vancouver (1757-1798), quien al término de su viaje de circunnavegación por el mundo, exploró la Isla del Coco (1795) (Arias 1993). Hay una isla ubicada al sureste llamada Vancouver o Juan Bautista. Actualmente, Bahía Chatham es el principal punto de anclaje en la isla, en ella también se encuentra una estación pequeña para los Guarda Recursos del Ministerio de Ambiente, Energía y Telecomunicaciones (MINAET) y las rocas donde numerosos viajeros han dejado constancia de su estadía en la isla. Chatham es también el nombre de una localidad portuaria en el sur de Inglaterra.

El Cerro Iglesias o Yglesias es la mayor elevación de la isla (635 m.s.n.m.) conformada por un volcán extinto. Se bautizó en honor del presidente Rafael Iglesias Castro (1861-1924), quien fue presidente de Costa Rica en dos períodos consecutivos (1894-1898 y 18981902) en cuya administración se organizaron dos viajes de exploración a la Isla del Coco en 1898 y 1902. Fue en este último año que empezó a escribir su apellido de la forma Yglesias. Cabe destacar que otros tres accidentes geográficos importantes, cómo lo son el Río Iglesias, la Cascada Iglesias y la Bahía Iglesias, también recibieron su nombre en honor a este gobernante de Costa Rica (Fischel 1992, Arias 1993, Díaz 2005).

Dentro de los topónimos correspondientes a la fauna que se encuentra en este territorio insular cabe resaltar la quebrada El Pinzón cuyo nombre proviene de un pinzón (Pnaroloxias inornata), endémico de la isla y junto con los trece pinzones de las Islas Galápagos, forma parte de los llamados pinzones de Darwin que fueron estudiados por el célebre naturalista inglés para establecer sus postulados sobre la evolución de las especies (Montoya 2007).

La quebrada Venado recibe su nombre de una de las seis especies de mamíferos que existen actualmente en la Isla y que fueron introducidas en el pasado por los seres humanos, intencional o accidentalmente. En la actualidad se debate si la presencia de estas especies constituye una verdadera amenaza para la biodiversidad de la isla (Montoya 2007). Agrega Montoya que para el venado cola blanca (Odocoieleus virginianus) existe una referencia que indica la introducción, de un macho y dos hembras, de este venado en junio de 1935, por miembros de la expedición del barco Veracity de búsqueda del tesoro de la Treasure Recovery Limited.

Cabe señalar que Salguero (1965), menciona la introducción de un grupo de cerdos (Sus scrofa) y una pareja de cabras (Capra hircus) en la isla por parte de la expedición del Cap. James Collnett (1753-1806) en 1793. La presencia de estos animales, así como la de los venados, permitía a los visitantes efectuar labores de cacería para obtener carne fresca: "Es frecuente observar desde los botes, cuando se recorren los alrededores de la isla, a los venados, cabros [sic] y cerdos, los cuales se quedan generalmente quietos, ofreciendo fáciles blancos al cazador acuático. Sin embargo, la fauna ha sido muy diezmada y ya no se encuentran las grandes manadas de que hablaron viejos cronistas".

Una de las actividades económicas más importantes que se desarrolla en la Isla del Coco es la del buceo como actividad turística. La mayoría de estos turistas son extranjeros y la visitan por ser su principal atractivo la observación de las diferentes especies de tiburón presentes en este Parque Nacional. La roca Aleta de Tiburón recibe su nombre debido a su semejanza con esta parte del animal tan abundante en los alrededores de la isla (Montoya com. pers. 2012). 
También, la experiencia durante este trabajo ha permitido rectificar algunos de los topónimos, entre ellos los cabos Atrevido y Descubierto, que deben llamarse Atrevida y Descubierta (Fig. 2), nombre de las dos corbetas de la expedición española liderada por Alessandro Malaspina (1754-1810), que en 1791 pasó por la Isla del Coco (Arias 1993, Montoya 2007).

\section{DISCUSIÓN}

Los resultados presentados en este trabajo, constituyen un esfuerzo multidisciplinario en donde convergen el aporte de científicos sociales, principalmente historiadores y científicos de las llamadas ciencias básicas, lo que demuestra la importancia que tienen los estudios multidisciplinarios para el conocimiento del origen de los topónimos, en este caso, los relacionados con los nombres de los accidentes geográficos presentes en la Isla del Coco, territorio insular costarricense en el Océano Pacífico oriental, debido a la diversidad de criterios que dieron origen al nombre de dichos sitios.

Dentro de esta iniciativa, la metodología empleada permitió la identificación de setenta y seis topónimos del Parque Nacional Isla del Coco, a partir de la consulta de diversas fuentes históricas, de las cuales, se han documentado setenta y uno de ellos. Los topónimos investigados pueden ser agrupados en cuatro grupos principales: 1) aquellos pertenecientes a los navegantes, científicos, exploradores y

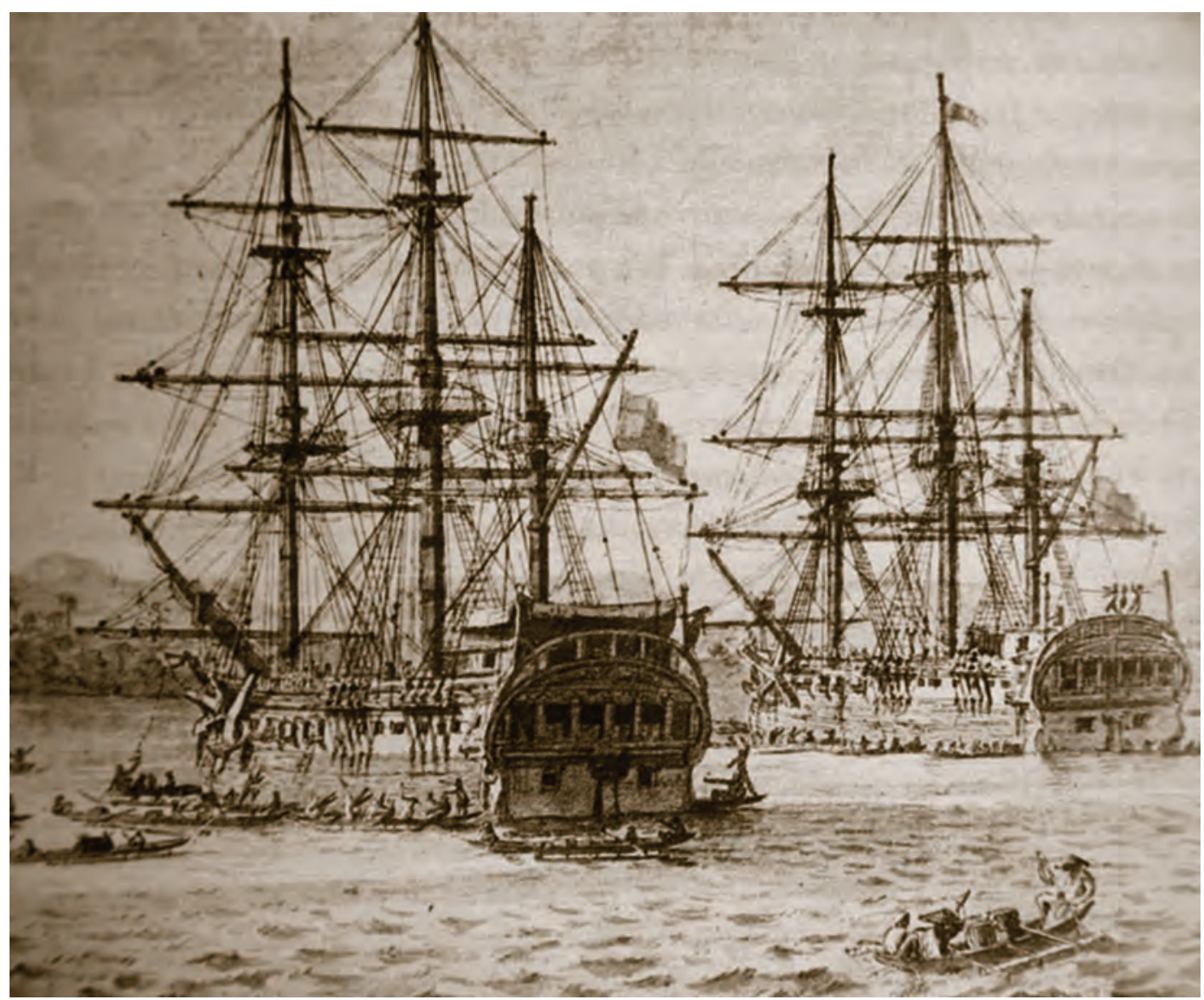

Fig. 2. Las corbetas Atrevida y Descubierta .Tomado del sitio público Wikimedia Commons.

Fig. 2. The corvettes Atrevida and Descubierta. Taken from the public site Wikimedia Commons. 
visitantes que arribaron a la isla entre los siglos XVII y XX, 2) los relacionados con actores políticos de la vida republicana de Costa Rica, historia de la isla, funcionarios del parque nacional y aspectos de índole temporal, 3) aquellos asociados con el relieve (geomorfología), 4) la flora y fauna, tanto terrestre como marina, que se encuentra en el Parque Nacional de la Isla del Coco (Cuadro 1 y Fig. 1). Sin embargo, hay todavía unos cinco topónimos cuyo origen no se ha podido ubicar, por lo que se requiere de mayor investigación e incluso presenta la posibilidad de incorporar fuentes orales de personas que han estado en contacto con la isla y esto podría arrojar alguna luz sobre la procedencia de esos nombres.

Al mismo tiempo, la confrontación con los mapas históricos, permitiría ubicar el período en que se fueron identificando los distintos topónimos y compararlos con otros mapas contemporáneos, entre ellos los relacionados con la batimetría y la práctica del buceo. Además, algunos topónimos tienen dos nombres, lo que podría evidenciar que no se trata de nombres oficiales sino de puntos de referencia dentro de la isla o bien, la existencia de un cambio en los mismos, aspecto que debe ser considerado en el producto final de la investigación.

Cabe destacar que debido a la naturaleza de este trabajo, el proyecto del Diccionario Histórico-Toponímico de la Isla del Coco, fue concebido en forma dinámica y la divulgación de sus resultados a través de la publicación de folletos, la elaboración de textos digitales en formato DVD y en páginas web, deberá tener implícito un proceso de retroalimentación que permita acopiar más datos relacionados con los topónimos presentes en dicho territorio insular. De esta forma, se podría ampliar y complementar la información con otras fuentes aún no localizadas en el desarrollo de la presente investigación. Este diccionario contribuye con el conocimiento de la toponimia de una porción del territorio costarricense que ha sido escasamente abordado por los estudios que se han realizado sobre los topónimos de Costa Rica.

\section{AGRADECIMIENTOS}

El presente trabajo constituye una reflexión sobre el proyecto "Diccionario Histórico-Toponímico de la isla del Coco" (VI-805-A9-742) que se ha venido ejecutando en el marco del Programa de Estudios Sociales de la Ciencia, la Técnica y el Medio Ambiente (PESCTMA, VI805-A4-906), adscrito al Centro de Investigaciones Geofísicas (CIGEFI) de la Universidad de Costa Rica. Además, este proyecto cuenta con el apoyo de los siguientes proyectos adscritos al Centro de Investigación en Ciencias del Mar y Limnología (CIMAR): “Generación de escenarios de cambio climático en la Isla del Coco, Corredor de Conservación Marina del Pacífico Tropical del Este" (VI-808-A9-180), "Interacciones Océano-Atmósfera y la Biodiversidad Marina de la Isla del Coco, Costa Rica" (VI-808-B0-654) y "Algunos aspectos de la dinámica de mesoescala del Pacífico Tropical Este (PTE) que pueden tener relación con la productividad alrededor de la Isla del Coco" (VI-808-B0-092). Los autores agradecen también a Alfonso Patricio Reinoso Gaguancela, miembro de la Casa de la Cultura Ecuatoriana "Benjamín Carrión” Núcleo del Cañar (Ecuador), y a María José Martínez Semanate de la Pontificia Universidad Católica del Ecuador, por facilitar textos referentes a la historia de las Islas Galápagos que han sido de gran utilidad para la presente investigación. Por último a Paula Pérez, por su ayuda en la elaboración del mapa presentado en la Figura 1 y a Michel Montoya por sus aportes y comentarios al manuscrito.

\section{RESUMEN}

Se recolectaron de distintas fuentes documentales los nombres de setenta y seis topónimos correspondientes a los principales accidentes topográficos del Parque Nacional Isla del Coco, Costa Rica. Estos se pueden agrupar en cuatro grupos principales, como son aquellos pertenecientes a las visitas de navegantes británicos, la vida republicana en Costa Rica y aquellos de la flora y fauna, tanto terrestre como marina. La metodología empleada ha permitido identificar setenta y uno de estos topónimos. Conceptualmente, el dinamismo del abordaje metodológico y la divulgación 
de sus resultados sugieren tener implícito un proceso de retroalimentación.

Palabras clave: toponimia, Isla del Coco, Pacífico Tropical Este, historia de Costa Rica, América Central, mapa.

\section{REFERENCIAS}

Alfaro, A. 1963. Flora y fauna. Memoria de la Secretaría de Fomento 1899. Reproducciones científicas, una expedición y legislación de la Isla del Coco. IGN. San José, Costa Rica: 31-36.

Ancey, C.F. 1903. Faune malacologique terrestre de 1'Ile des Cocos dans I'Ocean Pacifique. J. Conchyl. 51: 97-104.

Arias, R. 1993. La Isla del Coco: perspectiva histórica y análisis de una leyenda. Tesis de Licenciatura en Historia, Univ. Costa Rica, San Pedro, Costa Rica. 200 p.

Arias, R. 2004. Análisis del patrimonio histórico cultural de Costa Rica como política pública: el caso de la Isla del Coco. Tesis de Maestría en Administración Pública, Univ. Costa Rica, San Pedro, Costa Rica. 204 p.

Azofeifa, E. 1986. Toponimia cantonal de Costa Rica. Aproximaciones. IFAM. San José, Costa Rica. 135 p.

Biolley, P. 1935. Moluscos de las Isla del Coco. Revista del Colegio de Señoritas (San José) 2(6): 2-18 (Original en francés: Biolley, P. 1907. Mollusques de l'Isla del Coco. Museo Nacional de Costa Rica. Tip. Nacional, San José, Costa Rica. 30 p.).

Boza, M. 1984. Guía de los Parques Nacionales de Costa Rica. Fundación de Parques Nacionales - Incafo, S.A., Madrid, España. 128 p.

Carbonell, M. 1998. Costa Rica designates Isla del Coco as its 7 th site. Parque Nacional Isla del Coco. Ramsar Convention. (Consultado: 30 septiembre 2010, http://www.ramsar.org/cda/en/ramsarnews-archives-1998-costa-rica-designates/main/ ramsar/1-26-45-91^20261_4000_0).

Chang, G. 2010. Toponimia de la provincia de Limón. MCJ - Impr. Nacional, San José, Costa Rica. 340 p.

Chavarría, N. 1963. Observaciones. Memoria de la Secretaría de Fomento 1899. Reproducciones científicas, una expedición y legislación de la Isla del Coco. IGN. San José, Costa Rica: 37-43.

Cortés, J. 2008. Historia de la investigación marina de la Isla del Coco, Costa Rica. Rev. Biol. Trop. 56 (Supl. 2): $1-18$.

Dampier, W. 1697. A New Voyage Round the World. James Knapton, Londres.

Díaz, D. 2005. Construcción de un Estado moderno. Política, Estado e identidad nacional en Costa Rica, 1821-1914. Edit. Univ. Costa Rica, San José, Costa Rica. 88 p.
Enríquez, F. 1998. Diversión pública y sociabilidad en las comunidades cafetaleras de San José: el caso de Moravia (1890-1930). Tesis de Maestría en Historia, Univ. Costa Rica, San Pedro, Costa Rica. 244 p.

Fernández, C. 2008. Flora marina del Parque Nacional Isla del Coco, Costa Rica, Pacífico Tropical Oriental. Rev. Biol. Trop. 56 (Supl. 2): 57-69.

Figueroa, J. 1900. Álbum de Figueroa. I y II. Archivos Nacionales, san José, Costa Rica.

Fischel, A. 1992. El Teatro Nacional de Costa Rica, su historia. Edit. Teatro Nacional, San José, Costa Rica. $393 \mathrm{p}$.

Gagini, C. 1979. Diccionario de costarriqueñismos. Edit. Costa Rica, San José, Costa Rica. 243 p.

Gallardo, H. 1991. Elementos de investigación académica. Edit. UNED, San José, Costa Rica. 239 p.

Garita, F. 1995. Toponimia de la provincia de Cartago. MCJD - Edit. Univ. Costa Rica, San José, Costa Rica.

Garita, F. 1998. Toponimia de la provincia de Heredia. Edit. IGN, San José, Costa Rica. 104 p.

Garita, F. 2000. Toponimia de la provincia de Guanacaste. IGN, San José, Costa Rica. 220 p.

Garita, F. \& J. Cordero. 2002. Toponimia de la provincia de Puntarenas. IGN, San José, Costa Rica. 352 p.

Guzmán, H.M. \& J. Cortés. 1992. Cocos Island (Pacific of Costa Rica) coral reefs after the 1982-83 El Niño disturbance. Rev. Biol. Trop. 40: 309-324.

Guzmán, H.M. \& J. Cortés. 1993. Los arrecifes coralinos del Pacífico Oriental Tropical: Revisión y perspectivas. Rev. Biol. Trop. 41: 535-557.

Guzmán, H.M. \& J. Cortés. 2007. Reef recovery 20-yr after the 1982-83 El Niño massive mortality. Mar. Biol. 151: 401-411.

Hernández, F. \& E. Bedoya. 2010. Toponimia de la provincia de Alajuela. Edit. Univ. Costa Rica, San José, Costa Rica. 326 p.

ICOMOS Costa Rica. sf. Sitios de patrimonio natural. (Consultado: 24 septiembre 2010, http:// www.icomoscr.org/content/index.php/ patrim-ambiental/70-sitios-patrimonio-natural).

Instituto Geográfico Nacional. 2002. Isla del Coco (mapa). Escala 1:25000. Proyección transversa de Mercator. IGN, San José, Costa Rica.

Lièvre, D. 1962. Una isla desierta en el Pacífico. La isla del Coco. J. León \& M. León (traductores). Los viajes de Cockburn y Lièvre por Costa Rica. Edit. Costa Rica, San José, Costa Rica: 7-134.

Ministerio de Obras Públicas y Transportes. 2009. Instituto Geográfico Nacional. División territorial administrativa de la República de Costa Rica. MOPT, San José, Costa Rica. 138 p. 
Monge, M.R., M.T. Bolaños, M. Fonseca, M. Zumbado \& A. Guillén. 2003. Toponimia de la provincia de San José. Edit. IGN, San José, Costa Rica. 284 p.

Montoya, M. 2007. Conozca la Isla del Coco. Una guía para su visitación. OET, San José, Costa Rica.

Moreno, A. s.f. Toponimia y análisis histórico. (Consultado: 6 junio 2011, http://codex.colmex.mx:8991/ exlibris/aleph/a18_1/apache_media/XS8R5TS7LQU8XKYVCB1TP2YHD42DYC.pdf).

Passmore, R. 1963. Informe sobre la isla del Coco. Memoria de la Secretaría de Fomento 1899. Reproducciones científicas, una expedición y legislación de la isla del Coco. San José, Costa Rica. IGN: 7-12.

Pittier, H. 1895. Nombres geográficos de Costa Rica. I. Talamanca (Primera contribución). Tipografía Nacional, San José, Costa Rica. 46 p.

Pittier, H. 1899. Apuntamientos preliminares sobre la Isla del Cocos, posesión costarricense en el Océano Pacífico. Memoria Secretaría de Fomento, San José, Costa Rica: 15-28.

Sáenz, J., J. Fernández \& M. Muñoz. 2001. Primeras Damas de Costa Rica. ICE, San José, Costa Rica. $760 \mathrm{p}$.

Salguero, M. 1965. Isla del Coco, huerto del Océano. In La Nación (Suplemento La Vida en Tiquicia). 24 de abril: $33-40$.
Segura, J. 1996. Costa Rica y su contexto internacional: Pp. 309-355. In J. Quesada (Ed.). Costa Rica contemporánea. Raíces del Estado de la Nación. Edt. Univ. Costa Rica, San José, Costa Rica.

Steinleger, C. 2004. Galápagos: objetivo militar de los EE.UU. NuestraAmérica.info (Consultado: 25 septiembre 2010, http://www.nuestraamerica.org/leer. hlvs/3592).

Sylva, P. 1992. Las islas Galápagos en la historia del Ecuador, p. 253-303. In Nueva historia del Ecuador (12). Ensayos generales I: Espacio, población, región. Corporación Editora Nacional - Grijalbo Ecuatoriana, Quito, Ecuador.

Ugalde, L. 1993. El derecho de Costa Rica sobre la Isla del Coco. Tesis de Licenciatura en Derecho, Univ. Costa Rica. 383 p.

Wafer. L. 1967. Un viaje a Costa Rica. Anales Acad. Geogr. Hist. Costa Rica 1965-1966: 65-78.

Weston, C. 1992. La Isla del Coco. Cocos Island. Edit. Trejos, San José, Costa Rica. 310 p.

Wikimedia Commons. 2010. Descubierta y Atrevida. (Consultada: 26 septiembre 2010, http://upload.wikimedia.org/wikipedia/commons/3/3b/Descubiertaatrevida.jpg). 
ANEXO 1

Lista de los topónimos identificados en la Isla del Coco, Costa Rica.

\section{APPENDIX 1}

List of toponymys identified at Isla del Coco, Costa Rica.

\begin{tabular}{|c|c|c|c|c|}
\hline Nombre & $\begin{array}{l}\text { Geoforma o } \\
\text { accidente } \\
\text { geográfico }\end{array}$ & Tipo & Característica & Origen \\
\hline Chatham $(3,9)$ & Bahía & Lembotopónimo & Embarcación & $\begin{array}{l}\text { Nave de la expedición de George } \\
\text { Vancouver (1795) }\end{array}$ \\
\hline $\begin{array}{l}\text { Iglesias (Yglesias) o } \\
\text { Esperanza }(2,3,9)\end{array}$ & Bahía & Epotopónimo & Actor político & $\begin{array}{l}\text { Rafael Iglesias: Presidente de Costa Rica } \\
\text { (1894-1902) }\end{array}$ \\
\hline Inútil (9) & Bahía & Fisiotopónimo & Geomorfología & $\begin{array}{l}\text { Lugar no adecuado para la instalación del } \\
\text { presidio }\end{array}$ \\
\hline Wafer $(3,8,9)$ & Bahía & Epotopónimo & Explorador & Lionel Wafer: Cirujano galés del siglo XVII \\
\hline Weston $(2,3,4,9)$ & Bahía & Epotopónimo & Explorador & $\begin{array}{l}\text { Julian Weston: Explorador y marino inglés } \\
\text { del siglo XX }\end{array}$ \\
\hline Alcyone $(2,9)$ & Bajo & Lembotopónimo & Embarcación & $\begin{array}{l}\text { Barco de la expedición de la Sociedad } \\
\text { Cousteau que visitó la isla en } 1987\end{array}$ \\
\hline Dos Amigos (9) & Bajo & Topotopónimo & Geomorfología & Situado cerca de las islas Dos Amigos \\
\hline Punta María (9) & Bajo & Actiónimo & Geomorfología & Véase Punta María \\
\hline Atrevida $(3,5,9)$ & Cabo & Lembotopónimo & Embarcación & $\begin{array}{l}\text { Navío de la expedición de Alessandro } \\
\text { Malaspina (1791) }\end{array}$ \\
\hline Barreto (9) & Cabo & Epotopónimo & Visitante & $\begin{array}{l}\text { Posiblemente por un visitante que llegó a la } \\
\text { isla a finales del siglo XIX }\end{array}$ \\
\hline $\begin{array}{l}\text { Dampier o Esquina } \\
\text { Manta }(3,5,9)\end{array}$ & Cabo & Epotopónimo & Navegante & $\begin{array}{l}\text { William Dampier: Navegante y explorador } \\
\text { inglés del siglo XVII }\end{array}$ \\
\hline Descubierta $(3,8,9)$ & Cabo & Lembotopónimo & Embarcación & $\begin{array}{l}\text { Navío de la expedición de Alessandro } \\
\text { Malaspina (1791) }\end{array}$ \\
\hline Discovery $(3,9)$ & Cabo & Lembotopónimo & Embarcación & $\begin{array}{l}\text { Nave de la expedición de George } \\
\text { Vancouver (1795) }\end{array}$ \\
\hline Lionel $(3,5,9)$ & Cabo & Epotopónimo & Explorador & Lionel Wafer: Cirujano galés del siglo XVII \\
\hline $\begin{array}{l}\text { Iglesias (Yglesias) } \\
(2,3,9)\end{array}$ & Cascada & Epotopónimo & Actor político & $\begin{array}{l}\text { Rafael Iglesias: Presidente de Costa Rica } \\
\text { (1894-1902) }\end{array}$ \\
\hline $\begin{array}{l}\text { Jesús Jiménez } \\
(3,4,7,9)\end{array}$ & Cerro & Epotopónimo & Actor político & $\begin{array}{l}\text { Jesús Jiménez: Presidente de Costa Rica } \\
(1863-1866 \text { y } 1868-1870)\end{array}$ \\
\hline $\begin{array}{l}\text { Iglesias (Yglesias) } \\
(2,3,9)\end{array}$ & Cerro & Epotopónimo & Actor político & $\begin{array}{l}\text { Rafael Iglesias: Presidente de Costa Rica } \\
\text { (1894-1902) }\end{array}$ \\
\hline Pelón (9) & Cerro & Fisiotopónimo & Geomorfología & $\begin{array}{l}\text { Producto de la vegetación secundaria } \\
\text { generada por un derrumbe natural }\end{array}$ \\
\hline Challe $(4,6,9)$ & Estrecho & Epotopónimo & Explorador & $\begin{array}{l}\text { André Challe: empresario y viajero } \\
\text { costarricense del siglo XX }\end{array}$ \\
\hline Esperanza (9) & Estrecho & Actiónimo & Geomorfología & $\begin{array}{l}\text { Por encontrarse entre la Bahía Iglesias } \\
\text { (Esperanza) y la isla Muela }\end{array}$ \\
\hline $\begin{array}{l}\text { Cáscara o Viquinga } \\
(2,9)\end{array}$ & Isla & Fitotopónimo & Flora & Cáscaras del fruto del palo de hierro \\
\hline
\end{tabular}


ANEXO 1 (Continuación)

Lista de los topónimos identificados en la Isla del Coco, Costa Rica.

APPENDIX 1 (Continued)

List of toponymys identified at Isla del Coco, Costa Rica.

\begin{tabular}{|c|c|c|c|c|}
\hline Nombre & $\begin{array}{l}\text { Geoforma o } \\
\text { accidente } \\
\text { geográfico }\end{array}$ & Tipo & Característica & Origen \\
\hline $\operatorname{Coco}(1,3,9)$ & Isla & Fitotopónimo & Flora & $\begin{array}{l}\text { Castellanización del término francés } \\
\text { Coques }\end{array}$ \\
\hline Cónico (9) & Isla & Fisiotopónimo & Geomorfología & Forma de cono \\
\hline Dos Amigos (9) & Isla & Fisiotopónimo & Geomorfología & $\begin{array}{l}\text { Dos pequeñas islas que se encuentran } \\
\text { próximas al sudoeste de la isla del Coco }\end{array}$ \\
\hline $\begin{array}{l}\text { Manuelita o Nuez } \\
(2,7,9)\end{array}$ & Isla & $\begin{array}{l}\text { Epotopónimo / } \\
\text { Fitotopónimo }\end{array}$ & $\begin{array}{l}\text { Actor político / } \\
\text { Flora }\end{array}$ & $\begin{array}{l}\text { Esposa del presidente Iglesias. Fruto del } \\
\text { palo de hierro }\end{array}$ \\
\hline $\begin{array}{l}\text { Muela o Taza de Té } \\
\text { (9) }\end{array}$ & Isla & Fisiotopónimo & Geomorfología & $\begin{array}{l}\text { Semejanza a una taza al revés o a una } \\
\text { muela con caries por la presencia de un } \\
\text { agujero }\end{array}$ \\
\hline Pájara (9) & Isla & Zootopónimo & Fauna terrestre & $\begin{array}{l}\text { Sitio de anidación de los piqueros o } \\
\text { alcatraces (Sula sula y Sula leucogaster) }\end{array}$ \\
\hline $\begin{array}{l}\text { Vancouver o Juan } \\
\text { Bautista }(3,4,5,9)\end{array}$ & Isla & Epotopónimo & Navegante & $\begin{array}{l}\text { Navegante y explorador inglés del siglo } \\
\text { XVIII }\end{array}$ \\
\hline $\begin{array}{l}\text { Llanos Palo de Hierro } \\
(8,9)\end{array}$ & Llanura & Fitotopónimo & Flora & $\begin{array}{l}\text { Nombre común del árbol (Sacoglottis } \\
\text { holdridge) }\end{array}$ \\
\hline Colnett $(1,2,3,9)$ & Península & Epotopónimo & Navegante & $\begin{array}{l}\text { James Colnett: capitán inglés del siglo } \\
\text { XVIII }\end{array}$ \\
\hline Agujas (9) & Punta & Fisiotopónimo & Geomorfología & La saliente de la Península Colnett \\
\hline Don Felipe (9) & Punta & Epotopónimo & Funcionario & $\begin{array}{l}\text { Felipe Avilés: Ex funcionario del parque } \\
\text { nacional }\end{array}$ \\
\hline Don Will (9) & Punta & Epotopónimo & Funcionario & $\begin{array}{l}\text { Wilfrido Cordero T. (Padre): Ex funcionario } \\
\text { del parque nacional }\end{array}$ \\
\hline Gissler $(1,2,3,4,8,9)$ & Punta & Epotopónimo & Explorador & $\begin{array}{l}\text { August Gissler: colonizador alemán de la } \\
\text { isla en los siglos XIX y XX }\end{array}$ \\
\hline Madriz (9) & Punta & Epotopónimo & Funcionario & $\begin{array}{l}\text { Walter Madriz: funcionario activo del } \\
\text { parque nacional }\end{array}$ \\
\hline María (9) & Punta & Lembotopónimo & Embarcación & $\begin{array}{l}\text { Barco Mary Dear del Capitán William } \\
\text { Thompson, a quien se atribuye el entierro } \\
\text { de uno de los supuestos tesoros de la isla }\end{array}$ \\
\hline Montealegre (9) & Punta & Epotopónimo & Actor político & $\begin{array}{l}\text { José María Montealegre: Presidente de } \\
\text { Costa Rica (1859-1863) }\end{array}$ \\
\hline Pacheco $(4,9)$ & Punta & Epotopónimo & Actor político & $\begin{array}{l}\text { Ricardo Pacheco: Subsecretario de Fomento } \\
\text { que firmó el contrato de colonización con } \\
\text { Gissler }\end{array}$ \\
\hline Presidio $(5,8,9)$ & Punta & Pragmatopónimo & $\begin{array}{l}\text { Historia de la Isla } \\
\text { del Coco }\end{array}$ & $\begin{array}{l}\text { Centro penal instalado en la isla en el siglo } \\
\text { XIX }\end{array}$ \\
\hline
\end{tabular}


ANEXO 1 (Continuación)

Lista de los topónimos identificados en la Isla del Coco, Costa Rica.

APPENDIX 1 (Continued)

List of toponymys identified at Isla del Coco, Costa Rica.

\begin{tabular}{|c|c|c|c|c|}
\hline Nombre & $\begin{array}{l}\text { Geoforma o } \\
\text { accidente } \\
\text { geográfico }\end{array}$ & Tipo & Característica & Origen \\
\hline Quirós (9) & Punta & Epotopónimo & Actor político & $\begin{array}{l}\text { General Pedro Quirós: comandó dos } \\
\text { expediciones a la isla del Coco (1880 y } \\
1882)\end{array}$ \\
\hline Rodríguez $(3,4,9)$ & Punta & Epotopónimo & Actor político & $\begin{array}{l}\text { José Joaquín Rodríguez: presidente de } \\
\text { Costa Rica (1890-1894) }\end{array}$ \\
\hline Sofía (9) & Punta & Epotopónimo & Funcionario & $\begin{array}{l}\text { Nombre de una funcionaria del Parque } \\
\text { Nacional Isla del Coco }\end{array}$ \\
\hline Turrialba $(3,8,9)$ & Punta & Lembotopónimo & Embarcación & $\begin{array}{l}\text { Embarcación de las expediciones } \\
\text { costarricenses de } 1902 \text { y } 1905\end{array}$ \\
\hline Ulloa $(4,9)$ & Punta & Epotopónimo & Actor político & $\begin{array}{l}\text { Juan José Ulloa: cónsul de Costa Rica en } \\
\text { Nueva York }\end{array}$ \\
\hline $\begin{array}{l}15 \text { de Setiembre } \\
(1,5,8,9)\end{array}$ & Quebrada & Pragmatopónimo & $\begin{array}{l}\text { Historia de la Isla } \\
\text { del Coco }\end{array}$ & $\begin{array}{l}\text { Fecha en la que Costa Rica tomó posesión } \\
\text { de la isla (1869) }\end{array}$ \\
\hline Albatros $(3,8,9)$ & Quebrada & Lembotopónimo & Embarcación & $\begin{array}{l}\text { Navío de la expedición de Alexander } \\
\text { Agassiz (1891) }\end{array}$ \\
\hline Alfaro $(3,5,9)$ & Quebrada & Epotopónimo & Científico & $\begin{array}{l}\text { Anastasio Alfaro: científico costarricense de } \\
\text { los siglos XIX y XX }\end{array}$ \\
\hline Alvarado $(3,7,9)$ & Quebrada & Epotopónimo & Actor político & $\begin{array}{l}\text { Luisa Alvarado: suegra del presidente } \\
\text { Rafael Iglesias }\end{array}$ \\
\hline Camarón $(8,9)$ & Quebrada & Zootopónimo & Fauna marina & $\begin{array}{l}\text { Crustáceo presente en la isla del Coco y } \\
\text { perteneciente a los géneros Archaeatya y } \\
\text { Macrobrachium }\end{array}$ \\
\hline Camila (9) & Quebrada & Epotopónimo & Funcionario & $\begin{array}{l}\text { Camila Figueroa: Hija del ex funcionario } \\
\text { Hugo Figueroa }\end{array}$ \\
\hline El Anolís (9) & Quebrada & Zootopónimo & Fauna terrestre & $\begin{array}{l}\text { Lagartija (Norops townsendi) endémica de } \\
\text { la isla }\end{array}$ \\
\hline El Cabro $(8,9)$ & Quebrada & Zootopónimo & Fauna terrestre & $\begin{array}{l}\text { Ganado caprino (Capra hircus) introducido } \\
\text { a finales del siglo XVIII }\end{array}$ \\
\hline El Cuclillo (9) & Quebrada & Zootopónimo & Fauna terrestre & $\begin{array}{l}\text { Cuclillo (Coccizus ferrugineus) endémico } \\
\text { de la isla }\end{array}$ \\
\hline El Gato $(8,9)$ & Quebrada & Zootopónimo & Fauna terrestre & $\begin{array}{l}\text { Felino (Felis catus) cuya presencia está } \\
\text { documentada desde finales del siglo XIX }\end{array}$ \\
\hline El Pinzón $(8,9)$ & Quebrada & Zootopónimo & Fauna terrestre & $\begin{array}{l}\text { Pinzón (Pnaroloxias inornata) que habita } \\
\text { en la isla }\end{array}$ \\
\hline Escorpión (9) & Quebrada & Zootopónimo & Fauna terrestre & $\begin{array}{l}\text { Escorpión (Opisthacanthus valeroi) } \\
\text { endémico de la isla }\end{array}$ \\
\hline Espíritu Santo $(8,9)$ & Quebrada & Zootopónimo & Fauna terrestre & Paloma (Gygis alba) que anida en la isla \\
\hline Haug (9) & Quebrada & Epotopónimo & Funcionario & $\begin{array}{l}\text { Germán Haug: funcionario del parque } \\
\text { nacional }\end{array}$ \\
\hline
\end{tabular}


ANEXO 1 (Continuación)

Lista de los topónimos identificados en la Isla del Coco, Costa Rica.

APPENDIX 1 (Continued)

List of toponymys identified at Isla del Coco, Costa Rica.

\begin{tabular}{|c|c|c|c|c|}
\hline Nombre & $\begin{array}{l}\text { Geoforma o } \\
\text { accidente } \\
\text { geográfico }\end{array}$ & Tipo & Característica & Origen \\
\hline Lièvre $(3,4,9)$ & Quebrada & Epotopónimo & Explorador & $\begin{array}{l}\text { Dominic Lièvre, comandante de Marina } \\
\text { francés del siglo XIX }\end{array}$ \\
\hline Minuto (9) & Quebrada & Cronotopónimo & Tiempo & $\begin{array}{l}\text { Tiempo que tarda atravesar el vado en su } \\
\text { desembocadura }\end{array}$ \\
\hline Pittier $(3,9)$ & Quebrada & Epotopónimo & Científico & $\begin{array}{l}\text { Henri Pittier: Científico suizo de los siglos } \\
\text { XIX y XX }\end{array}$ \\
\hline Venado $(8,9)$ & Quebrada & Zootopónimo & Fauna terrestre & $\begin{array}{l}\text { Venado de cola blanca (Odocoieleus } \\
\text { virginianus) introducido en } 1935\end{array}$ \\
\hline Genio $(1,3,9)$ & Río & Lembotopónimo & Embarcación & $\begin{array}{l}\text { Embarcación Le Génie de la expedición } \\
\text { francesa de } 1846\end{array}$ \\
\hline $\begin{array}{l}\text { Iglesias (Yglesias) } \\
(2,3,9)\end{array}$ & Río & Epotopónimo & Actor político & $\begin{array}{l}\text { Rafael Iglesias: Presidente de Costa Rica } \\
\text { (1894-1902) }\end{array}$ \\
\hline Aleta de Tiburón (9) & Roca & Fisiotopónimo & Geomorfología & $\begin{array}{l}\text { La parte saliente de la roca se asemeja la } \\
\text { aleta de un tiburón }\end{array}$ \\
\hline Bote (9) & Roca & Fisiotopónimo & Geomorfología & Forma de la roca \\
\hline Gissler $(1,2,3,4,8,9)$ & Roca & Epotopónimo & Explorador & $\begin{array}{l}\text { August Gissler: colonizador alemán de la } \\
\text { isla en los siglos XIX y XX }\end{array}$ \\
\hline Pan de Azúcar (9) & Roca & Fisiotopónimo & Geomorfología & Forma similar al cerro homónimo de Brasil \\
\hline Sucia (9) & Roca & Fisiotopónimo & Geomorfología & $\begin{array}{l}\text { Color negruzco producido por el } \\
\text { excremento de las aves }\end{array}$ \\
\hline Sumergida (9) & Roca & Fisiotopónimo & Geomorfología & Emerge cuando la marea es muy baja \\
\hline $\begin{array}{l}\text { Rocas Barlovento } \\
(8,9)\end{array}$ & Roca & Fisiotopónimo & Geomorfología & $\begin{array}{l}\text { Rocas situadas al suroeste de la isla, la } \\
\text { cual es la dirección predominante de donde } \\
\text { viene el viento }\end{array}$ \\
\hline
\end{tabular}

Fuentes: (1) Lièvre 1962, (2) Weston 1992, (3) Arias 1993, (4) Arias 2004, (5) Ugalde 1993, (6) Enríquez 1998, (7) Sáenz Fernández y Muñoz 2001, (8) Montoya 2007 y (9) Montoya com. pers. 2012. 